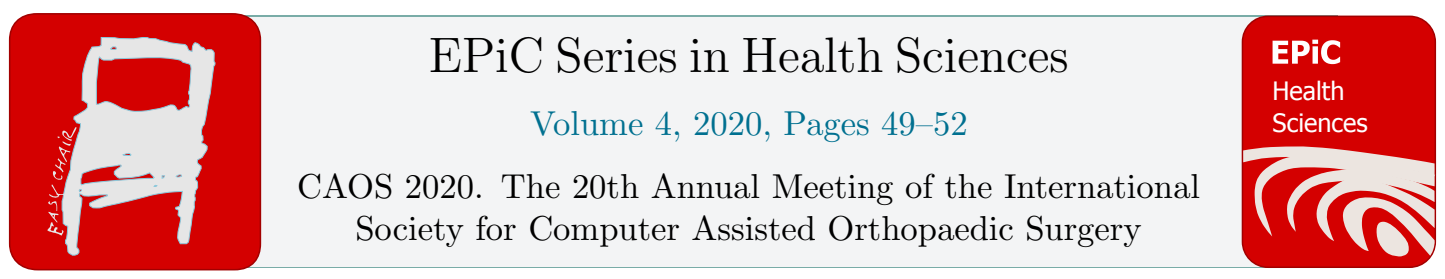

\title{
Total Knee Arthroplasty with CAOS Augmentation
}

\author{
Yifei Dai ${ }^{1 *}$, Amaury Jung ${ }^{2}$, Guillaume Bras $^{2}$, Ian Gradisar ${ }^{3 *}$ \\ ${ }^{1}$ Exactech Inc, Gainesville, FL 32653, USA \\ ${ }^{2}$ Blue Ortho, Gieres, FR \\ ${ }^{3}$ Crystal Clinic Orthopaedic Center, Akron, OH \\ iangradisar@gmail.com, Yifei.dai@exac.com
}

\begin{abstract}
This study sought to evaluate the efficiency, usage, and accuracy of a novel technology that augments mechanical instrumentation with intraoperative CAOS guidance. Technical reports on 411 primary TKA cases performed using the technology were reviewed. The results demonstrated high surgical efficiency (time) and resection accuracy (alignment and resection depth). Furthermore, it was observed that one fifth of the time, the surgeons placed the cutting block that deviated more than $>2 \% \mathrm{~mm}$ from the ideal position in the coronal plane. Substantial adjustments were found to be required (on average $\sim 10 \% \mathrm{~mm}$ per case) to correct the initial placement of the cutting block. The CAOS augmentation minimized the error in cutting block placement and assisted in achieving high accuracy in bony resections. The findings revealed the prevalence of clinical error with manual conventional bony preparation that can be addressed with efficiency and accuracy by adding CAOS augmentation to the mechanical instrumentation.
\end{abstract}

\section{Introduction}

Computer-assisted orthopedic surgery (CAOS) offers increased accuracy to total knee arthroplasty (TKA) bony resections compared to the conventional techniques [1]. Despite the proven benefits, one of the drawbacks for the adoption of CAOS technology may be the inconvenience of switching from conventional instruments to CAOS-specific instruments. A recent technology added CAOS augmentation to conventional mechanical instruments, removing the need for significant instrument learning. The system has been shown to have minimal learning curve [2] and offer good usability and demonstrated to be non-disruptive to the surgical flow during its early adoption, reported by a subjective survey by the users [3]. 
As the clinical applications of this CAOS technology continues, further assessment is desired to confirm the promising results under clinical setting. The purpose of this study was to collect and assess quantitative results regarding the use of the CAOS augmented technology based on the combined experiences of a pilot surgeon group.

\section{Materials and Methods}

A retrospective review was carried out on the technical records of 411 primary TKA cases (no patient information was reviewed). All cases were performed by a group of three surgeons using conventional mechanical instrumentation augmented by the CAOS technology. Three analyses were performed on the technical records regarding:

Efficiency of the CAOS augmented technology: The time spent on landmark acquisition and CAOS guidance, as well as the total CAOS guided surgical time were assessed.

Usage and scope of intraoperative CAOS guidance: The location of the initially placed tibial and femoral cutting blocks were reviewed along with the subsequent CAOS guided adjustment performed by the surgeon. The percentage of cases with cutting block initially placed at greater than $2^{\circ}$ varus/valgus were calculated. The total "clicks" for cutting block adjustment (coronal/sagittal alignments and resection depth) were assessed. Each "click" corresponded to the number of discrete changes surgeon tuned in on the resection guides ( 1 click corresponds to $1 \% \mathrm{~mm}$ adjustment increment on the instrument).

Accuracy of the bony resection: The intraoperatively measured deviations between the surgeon set resection goal and the achieved bony resection (coronal and sagittal alignments and resection depth) were calculated to investigate resection accuracy.

\section{Results}

On average, it took 1 minute or less for the acquisition of the anatomic landmarks on the tibia and femur, respectively (Table 1A). Similar results were found in the time spent on CAOS guided tibial and femoral cutting block adjustments. The total CAOS guided surgical time was found to be $11.1 \pm 4.4$ minutes per case, with $97.0 \%$ and $99.1 \%$ of the cases required no more than 20 minutes and 30 minutes, respectively.

In $19.8 \%$ and $22.4 \%$ of the cases, the initial cutting block placements had more than $2^{\circ}$ error in varus/valgus alignment, respectively for tibia and femur. The percentage reduced drastically to $\sim 1 \%$ after the surgeon adjusting the cutting block based on the CAOS guidance. The scope of adjustment "clicks" for all 411 TKA cases was summarized in Table 1B. Combining the adjustment "clicks" required for all three resection parameters (coronal and sagittal alignments and resection depth), on average each knee required approximately 10 "clicks" $(\% / \mathrm{mm})$ to correct the cutting block position. Significantly more "clicks" were needed to adjust the position of the femoral cutting block compared to tibial cutting block ( $\mathrm{p}<0.01$ ), with the difference found to locate in the adjustment of the sagittal alignment ( $<$ 0.01). No significant difference was found regarding the number of "clicks" between coronal alignment, sagittal alignment, and resection depth. High resection accuracy was achieved for both tibial and femoral resections (Table 1C). 


\section{Discussion}

This study reported the prevalence of inaccuracy in the position of manually placed cutting blocks during conventional TKA surgeries. It was observed that $20 \%$ of the time, the surgeons placed the cutting with more than $>2^{\circ}$ deviation from the ideal coronal alignment, potentially impacting clinical results [4]. Adding CAOS augmentation to mechanical instrumentation was demonstrated to sustainably improve resection accuracy. With CAOS guidance, considerable adjustments were required (on average a combined amount of $10 \% \mathrm{~mm}$ per case) to correct the cutting blocks to the proper position. Significantly more adjustment "click" was found in femoral flexion/extension compared to tibial posterior slope. This might be caused by the need to correct the intramedullary reamer established flexion/extension alignment to the mechanical axis, especially with the prevalence of femoral sagittal bowing. The results also demonstrated minimal impact on the surgical efficiency (time) from incorporating the CAOS augmentation into the surgical workflow.

With previous studies reported only $70 \%$ of conventional TKAs could achieve acceptable alignment accuracy [5,6], the findings from this study confirmed and emphasized the clinical need for mitigating error during manual conventional bony preparation, which can be addressed efficiently and accurately with CAOS augmented mechanical instrumentation.

\begin{tabular}{lc} 
A & \\
\hline $\begin{array}{l}\text { Landmark Acquisiton (min) } \\
\text { Tibia }\end{array}$ & $0.7 \pm 0.4$ \\
$\quad$ Femur & $1.0 \pm 1.0$ \\
& \\
CAOS Guided Cutting Block & \\
Adjustment (min) & $1.2 \pm 1.5$ \\
$\quad$ Tibia & $1.1 \pm 1.5$ \\
$\quad$ Femur & $11.1 \pm 4.4$ \\
Total Time for CAOS Surgery (min) & 97.0 \\
\%cases within 20 min & 99.1 \\
\hline \%cases within 30 min &
\end{tabular}

\begin{tabular}{lcc} 
C & & \\
\hline & Tibia & Femur \\
Signed Error & & \\
$\quad$ Alignment $\left({ }^{\circ}\right)$ & $0.0 \pm 1.3$ & $0.1 \pm 1.1$ \\
$\quad$ Resection Depth (mm) & $-0.2 \pm 1.4$ & $0.2 \pm 1.3$ \\
Unsigned (Absolute) Error & & \\
$\quad$ Alignment $\left(^{\circ}\right)$ & $1.0 \pm 0.9$ & $0.8 \pm 0.7$ \\
$\quad$ Resection Depth (mm) & $1.1 \pm 1.0$ & $1.0 \pm 0.8$ \\
\hline
\end{tabular}

\begin{tabular}{|c|c|c|c|c|}
\hline N Adjustment "Clicks" & Per Knee & Tibia & Femur & $\mathrm{P}$ (Tibia vs Femur) \\
\hline Coronal Alignment & $3.3 \pm 3.0$ & $1.7 \pm 2.4$ & $1.7 \pm 1.8$ & N.S. \\
\hline Sagittal Alignment & $3.6 \pm 3.0$ & $1.6 \pm 1.8$ & $2.1 \pm 2.4$ & $<0.01$ \\
\hline Resection Depth & $3.5 \pm 3.7$ & $1.7 \pm 2.3$ & $1.8 \pm 2.3$ & N.S. \\
\hline All Parameters Combined & $9.8 \pm 4.2$ & $4.5 \pm 2.6$ & $5.3 \pm 3.4$ & $<0.01$ \\
\hline
\end{tabular}

Table 1. A) efficiency, B) resection accuracy, and C) scope of CAOS guided adjustments in the TKA cases performed with CAOS augmented mechanical instrumentation. 

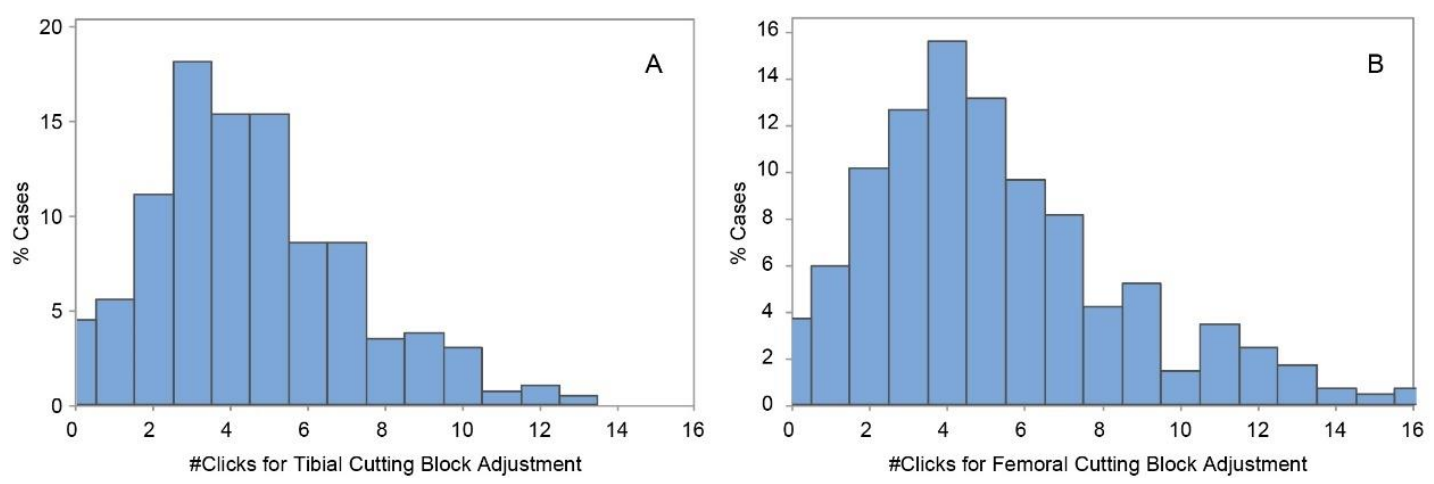

Figure 1. Percentage distribution of cases regarding the number of "clicks" $(\% / \mathrm{mm}$ adjustment $)$ required to correct the position of initially placed A) tibial and B) femoral cutting blocks.

\section{References}

[1] Sparmann M, et al. Positioning of total knee arthroplasty with and without navigation support: a prospective, randomised study. J Bone Joint Surg Br. 2003;85(6):830-5.

[2] Dai Y, et al. learning of a caos enhanced mechanical instrument system for total knee arthroplasty: a cusum analysis. CAOS 2018

[3] Dai Y, et al. Early experience with CAOS enhanced total knee arthroplasty - a global, multi-surgeon evaluation. CAOS 2019

[4] Walker PS, Meere PA, Bell CP. Effects of surgical variables in balancing of total knee replacements using an instrumented tibial trial. Knee. 2014;21(1):156-61

[5] Ritter MA, et al. Preoperative malalignment increases risks of failure after total knee arthroplasty. $\mathrm{J}$ Bone Joint Surg Am. 2013;95(2):126-31.

[6] Hetaimish BM, et al. Meta-analysis of navigation vs conventional total knee arthroplasty. J Arthroplasty. 2012;27(6):1177-82. 